\title{
Impedance and loss factor of a coaxial liner with many holes: Effect of the attenuation
}

\author{
A. Mostacci \\ Universitá di Roma “La Sapienza,” Roma, Italy \\ and CERN, Geneva, Switzerland \\ L. Palumbo \\ Universitá di Roma "La Sapienza," Roma, Italy \\ and INFN, Laboratori Nazionali di Frascati, C.P. 13, 00044 Frascati, Italy \\ F. Ruggiero \\ CERN, Geneva, Switzerland \\ (Received 25 August 1999; published 9 December 1999)
}

\begin{abstract}
In the framework of the modified Bethe's diffraction theory, we study the energy lost by a relativistic particle beam traveling in a coaxial liner with many holes, including the effect of attenuation in the coaxial region. The interference among the holes is the main source of losses and is affected by the attenuation in the coaxial only over sufficiently long distances. We derive analytical formulas for all the interesting quantities and particular attention is given to clarifying the physical meaning of the results; numerical examples are considered using LHC-like parameters.
\end{abstract}

PACS numbers: 41.75.-i, 41.20.-q

\section{INTRODUCTION}

Several papers have been devoted to the study of the interaction between a particle beam and the pumping holes, in order to get the coupling impedance (for a review see Refs. $[1,2])$. Of particular interest is the structure sketched in Fig. 1, where the holes couple the vacuum chamber (a circular waveguide) to the external antechamber (a coaxial waveguide), as in the LHC liner.

The problem has been solved by means of the modified Bethe's diffraction theory [3] for a single hole in [4] and for $N$ holes in [5], at low frequency. Recently, this theory has been further modified to estimate analytically the effects of a long narrow slot [6]. Some measurements were also performed on a LHC vacuum chamber prototype [7] (i.e., a coaxial chamber with many holes) to estimate the power lost by the beam, the results being explained by a simplified model. Other techniques often used are the field matching method [8] and a variational approach [9].

In this paper, we extend previous results of [5], including the effect of field attenuation in the coaxial region. A possible source of attenuation is the Ohmic loss

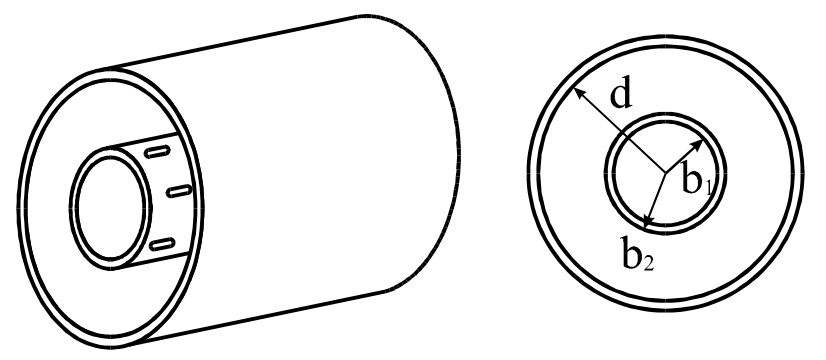

FIG. 1. Relevant geometries.

$1098-4402 / 99 / 2(12) / 124401(6) \$ 15.00$ in the walls of the coaxial, but some other source could be foreseen as well (as for instance, proper attenuators). We will focus mainly on the first case, but our theory can be easily extended to other cases. The energy dissipation is dominated by interference effects among the holes. The latter takes place only over lengths shorter than the "attenuation length" defined in Sec. V; accounting merely for interference effects would lead to the unphysical result of a diverging power loss per unit length. We show that for longer lengths of the perforated screen the attenuation plays a fundamental role, leading to a finite asymptotic value of the specific power loss.

For simplicity, we study the ideal structure of a pipe with thin walls $\left(b_{1}=b_{2}=b\right)$. After an outline of the general theory (Sec. II), in Sec. III we present the results for the coupling impedance in the case of attenuation in the coaxial. Since it has been demonstrated [5] that the loss factor is not significantly influenced by the randomization of the position of the holes, we focus on equally spaced holes (with one hole per cross section). Then we consider the attenuation due to Ohmic losses, discussing the loss factor in Sec. IV and the power lost per unit length in Sec. V. In Sec. VI we compare our results to those of Ref. [7], showing that they are in substantial agreement, and in Sec. VII we present our conclusions.

\section{OUTLINE OF THE GENERAL THEORY}

The general theory adopted in these calculations is described in [4,5]; for convenience, we summarize its main features at frequencies below the beam pipe cutoff considering only scattered TEM-type fields in the coaxial region.

(C) 1999 The American Physical Society

124401-1 
We will adopt the cylindrical coordinates, with $z$ being the longitudinal coordinate, $r$ and $\varphi$ the radial and the azimuthal coordinates, respectively. The subscript " 0 " on a field means that it is a source field (charge field), while the fields with subscript " $s$ " are the scattered fields propagating in the coaxial region. $N$ is the number of the holes in the structure.

The modified Bethe's diffraction theory [3] states that each hole is equivalent to an electric and a magnetic dipole whose moments are given by

$$
\begin{aligned}
M_{\varphi}\left(z_{i}\right) & =\alpha_{m}\left[H_{0 \varphi}\left(z_{i}\right)-H_{s \varphi}\left(z_{i}\right)\right], \\
P_{r}\left(z_{i}\right) & =\varepsilon \alpha_{e}\left[E_{0 r}\left(z_{i}\right)-E_{s r}\left(z_{i}\right)\right], \quad i=1, \ldots, N,
\end{aligned}
$$

where $\alpha_{m}$ and $\alpha_{e}$ are the polarizabilities of the hole and $H_{s \varphi}$ and $E_{s r}$ are the scattered fields calculated at the center of the hole with longitudinal coordinate $z_{i}$. The primary field on the aperture, generated by an ultrarelativistic point charge $q$, traveling along the axis of a perfectly conducting circular beam pipe of radius $b$, is $\left(k_{0}=\omega / c\right)$

$$
H_{0 \varphi}\left(z_{i}\right)=\frac{q}{2 \pi b} e^{-j k_{0} z_{i}}, \quad E_{0 r}\left(z_{i}\right)=Z_{0} \frac{q}{2 \pi b} e^{-j k_{0} z_{i}},
$$

where $Z_{0}$ is the characteristic impedance of vacuum.

In [5], the TEM-type scattered fields are assumed to have the same phase velocity of the charge. We consider here a complex propagation constant of the form

$$
k_{c}=k_{0}-j \alpha,
$$

where $\alpha$ is the attenuation constant. Both $k_{0}$ and $\alpha$ are given functions of the frequency $\omega$ with dimensions of $\left[\mathrm{m}^{-1}\right]$. If the attenuation constant is a real quantity, the field will be exponentially damped along the propagation direction ( $z$ axis); the source of this attenuation fixes the $\omega$ dependence of $\alpha$. On the other hand, a purely imaginary $\alpha$ takes into account a slowing down of the TEM fields in the external coaxial region; that could be, for example, the effect of a long series of uniformly spaced holes acting as the periodic perturbation of boundary conditions in any slow-wave device.

In general, the scattered fields can be expressed as a superposition of modes. The coefficients of the modal expansion are determined through the Lorentz reciprocity principle [3]; they are linear functions of the equivalent dipole moments of the apertures which can be obtained solving a $2 N \times 2 N$ linear inhomogeneous system.

Once the equivalent dipole moments have been determined, we can calculate the longitudinal coupling impedance, using the result [5]

$$
Z(\omega)=j \frac{\omega Z_{0}}{2 \pi q b} \sum_{i=1}^{N}\left[\frac{M_{\varphi}\left(z_{i}\right)}{c}+P_{r}\left(z_{i}\right)\right] e^{j k_{0} z_{i}} .
$$

The longitudinal coupling impedance represents the Green function of the problem; the loss factor depends on its real part, since $[10,11]$

$$
k(\sigma)=\frac{1}{\pi} \int_{0}^{\infty} Z_{\operatorname{Re}}(\omega) e^{-(\omega \sigma / c)^{2}} d \omega,
$$

for a Gaussian bunch of rms length $\sigma$.

The TEM mode in the coaxial region will dissipate some power on the walls, possibly causing undesired effects; we introduce the dissipated power per unit length $P$, expressed in terms of the loss factor as

$$
P=\frac{c Q^{2} k(\sigma)}{S_{b} L_{d}},
$$

where $Q$ is the bunch charge, $S_{b}$ is the bunch separation, $L_{d}$ is the device length, and $k(\sigma)$ is the loss factor due to the $N$ holes in the device.

\section{LONGITUDINAL COUPLING IMPEDANCE OF $N$ HOLES}

The $2 N \times 2 N$ linear system for dipole moments could be dealt with by means of the standard analytical and numerical techniques. However, as long as the energy radiated trough the holes is a minor fraction of the total incident energy, the scattered field can be considered as a small perturbation with respect to the primary field, and the system for $M_{\varphi}, P_{r}$ can be treated with perturbative procedures. The simplest approach is the iterative solution stopped at first order [5]. Thus, using the approximate dipole moments in Eq. (4) and considering uniformly spaced holes with distance $D$, one gets for the imaginary and the real part of the coupling impedance the following relations [12]:

$$
Z_{\operatorname{Im}}(\omega)=Z_{0} \frac{\omega}{4 \pi^{2} b^{2} c}\left[N\left(\alpha_{m}+\alpha_{e}\right)-\frac{\omega}{4 \pi b^{2} \ln (d / b) c}\left(\alpha_{m}-\alpha_{e}\right)^{2} \sum_{h=1}^{N-1}(N-h) e^{-\alpha h D} \sin \left(2 h \frac{\omega}{c} D\right)\right],
$$

and

$$
Z_{\mathrm{Re}}(\omega)=Z_{0} \frac{\omega^{2}}{16 \pi^{3} b^{4} \ln (d / b) c^{2}}\left[N\left(\alpha_{m}^{2}+\alpha_{e}^{2}\right)+\left(\alpha_{m}+\alpha_{e}\right)^{2} F(D, N, \alpha)+\left(\alpha_{m}-\alpha_{e}\right)^{2} B(D, N, \alpha, \omega)\right],
$$

where

$$
F(D, N, \alpha)=\sum_{h=1}^{N} \sum_{k=1}^{h-1} e^{\alpha\left(z_{k}-z_{h}\right)}=\sum_{h=1}^{N} \sum_{k=1}^{h-1} e^{\alpha(k-h) D}
$$


and

$$
B(D, N, \alpha, \omega)=\sum_{h=1}^{N-1}(N-h) e^{-\alpha h D} \cos \left(2 h \frac{\omega}{c} D\right) .
$$

It is worth noting that $Z_{\mathrm{Im}}$ has a dominant term independent of the position of the holes and equal to $N$ times the impedance of a single hole. The attenuation does not play an important role in this case. Its effect is more remarkable on the real part, $Z_{\mathrm{Re}}$, since the latter strongly depends on the spacing among the holes, as already shown in [5].

In Eq. (8), the first term $\left[\propto N\left(\alpha_{m}^{2}+\alpha_{e}^{2}\right)\right]$ is the contribution of noninteracting holes, while the terms weighted by $F(D, N, \alpha)$ and $B(D, N, \alpha, \omega)$ are due to the forward and backward propagating TEM waves.

The weights of $F$ and $B$ depend on the polarizabilities; the magnetic and the electric polarizabilities have opposite signs and for circular holes $\left|\alpha_{m}\right|$ and $\left|\alpha_{e}\right|$ differ by a factor of $2\left(\alpha_{m}=4 / 3 R^{3}\right.$ and $\alpha_{e}=-2 / 3 R^{3}$, where $R$ is the hole radius), while for long slots they can be very similar (slot much longer than its width) [12]. Thus we expect that for rectangular or rounded-end slots the contribution of backward propagating waves is more relevant than for circular holes.

The forward TEM wave, traveling in phase with the beam, causes most of the energy losses; for this reason, the term $\propto F$ is often the only one considered (see, for example, [7]).

Performing the sums that define $F(D, N, \alpha)$, we get

$F(D, N, \alpha)=\frac{N\left(e^{\alpha D}-1\right)+\left(e^{-\alpha D N}-1\right) e^{\alpha D}}{\left(e^{\alpha D}-1\right)^{2}}$.

If the spacing of the pumping holes is small compared to the attenuation length of the field (namely, $\alpha D \ll 1$ ) and for $N \gg 1$, Eq. (11) becomes

$$
F(D, N, \alpha) \simeq \frac{N}{\alpha D}+\frac{e^{-\alpha D N}-1}{(\alpha D)^{2}} ;
$$

this is the case, for instance, of the LHC [13] in which that relation holds for all the frequencies in the bunch spectrum.
On the other hand, $B$ has resonance peaks at frequencies $f_{n}=n c /(2 D)$ whose width depends on the attenuation constant and the height is proportional to $N^{2}$ [12]. If $D$ is small with respect to $\sigma$, the frequencies of the peaks are beyond the relevant part of the bunch spectrum. Far from the peaks and for large $N, B$ can be approximated by a constant [12],

$$
B(D, N, \alpha, \omega) \simeq-\frac{N}{2},
$$

showing that the backward propagating TEM wave has an effect at all frequencies.

Using Eqs. (12) and (13) in Eq. (8) yields

$$
\begin{aligned}
Z_{\mathrm{Re}}(\omega) \simeq & Z_{0} \frac{\omega^{2}\left(\alpha_{m}+\alpha_{e}\right)^{2}}{16 \pi^{3} b^{4} \ln (d / b) c^{2}} \\
& \times\left[\frac{N}{2}+\frac{N}{\alpha D}+\frac{e^{-\alpha D N}-1}{(\alpha D)^{2}}\right] .
\end{aligned}
$$

\section{LOSS FACTOR}

With no dissipation in the coaxial, when $D / \sigma<1$ and $N \gg 1$ one gets for the loss factor [12]

$$
k(\sigma) \simeq \frac{Z_{0} \sqrt{\pi} c}{128 \pi^{4} b^{4} \ln (d / b) \sigma^{3}} N^{2}\left(\alpha_{m}+\alpha_{e}\right)^{2},
$$

showing the importance of the interference effects between the holes due to the presence of the TEM field in the coaxial region. These are not always considered in the literature and cause the total loss factor to depend on $N^{2}$.

The previous Eq. (14) for the impedance holds for an attenuation source in the coaxial region such that the field propagating there remains essentially TEM shaped; this is, for instance, the case of distributed dielectric losses. In first approximation, the effect of lossy walls can also be treated in this way by using the following attenuation constant [7]:

$$
\alpha(\omega)=a \sqrt{\omega} \quad \text { with } \quad a=\frac{1}{2 Z_{0} \ln (d / b)}\left(\frac{\sqrt{\rho_{b}}}{b}+\frac{\sqrt{\rho_{d}}}{d}\right) \sqrt{\frac{\mu}{2}},
$$

where $\rho_{b}\left(\rho_{d}\right)$ is the resistivity of the cylindrical surface of radius $b(d)$. Although it is only an approximation, this approach is widely used in the estimation of losses in high power rf transmission lines [14].

Using Eq. (16) in Eq. (18) yields the real part of the coupling impedance for this particular source of attenuation, and then performing the integral of Eq. (5) we get the loss factor. This can be done analytically only under the approximations discussed earlier, namely, Eq. (14), yielding

$$
k(\sigma)=\frac{Z_{0}\left(\alpha_{m}+\alpha_{e}\right)^{2}}{16 \pi^{4} b^{4} \ln (d / b) c^{2}}\left[\frac{\sqrt{\pi}}{8}\left(\frac{c}{\sigma}\right)^{3} N+\frac{1}{2} \Gamma\left(\frac{5}{4}\right) \frac{c^{5 / 2}}{a D \sigma^{5 / 2}} N+\frac{I}{a^{2} D^{2}}\right],
$$

where $I$ is defined in Appendix A. In the limit of large $N(a N D \sqrt{c / \sigma} \gg 1)$, the third term in the square brackets becomes negligible with respect to the other two. 


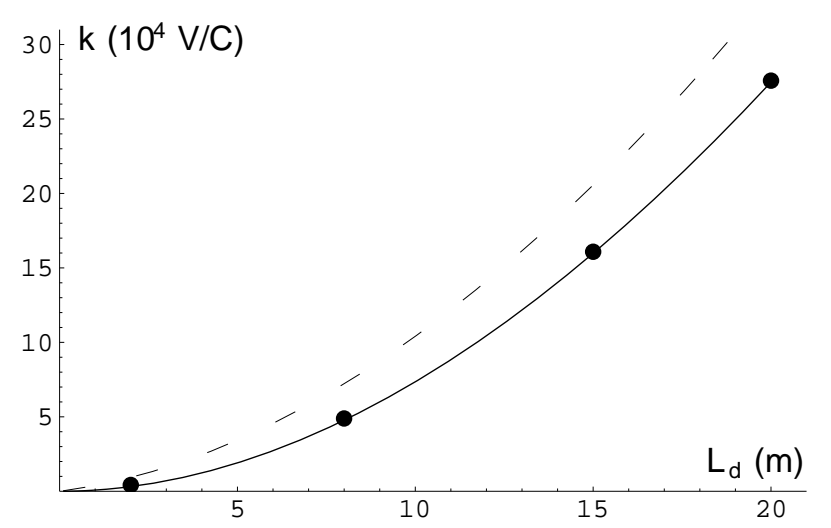

FIG. 2. Loss factor $k$ as a function of the length of the perforated beam screen $L_{d}$ (for a device length $L_{d}$ much shorter than the attenuation length $L_{\alpha} \simeq 80 \mathrm{~m}$ ). The solid line refers to Eq. (17) and the dots give the exact numerical values from Eq. (8). The dashed line is obtained neglecting backward wave. We use the following parameters: $b=19 \mathrm{~mm}, d=$ $24.5 \mathrm{~mm}, \rho_{b}=\rho_{d}=7.1 \times 10^{-7} \Omega / \mathrm{m}, D=16 \mathrm{~mm}, \sigma=$ $75 \mathrm{~mm}$, rounded end slots $8 \mathrm{~mm} \times 1.5 \mathrm{~mm}$.

The behavior of the loss factor as a function of the length of perforated screen $L_{d}=N D$ is shown in Figs. 2 and 3. When $L_{d}$ (or $N$ ) is small, the holes interfere and the loss factor grows quadratically as in the case of no attenuation (Fig. 2). However, as the length increases, not all the holes interact with each other because of the attenuation of the field. Thus, for large lengths the loss factor grows only linearly, as shown in Fig. 3. In both figures the dashed line is the loss factor obtained neglecting the backward

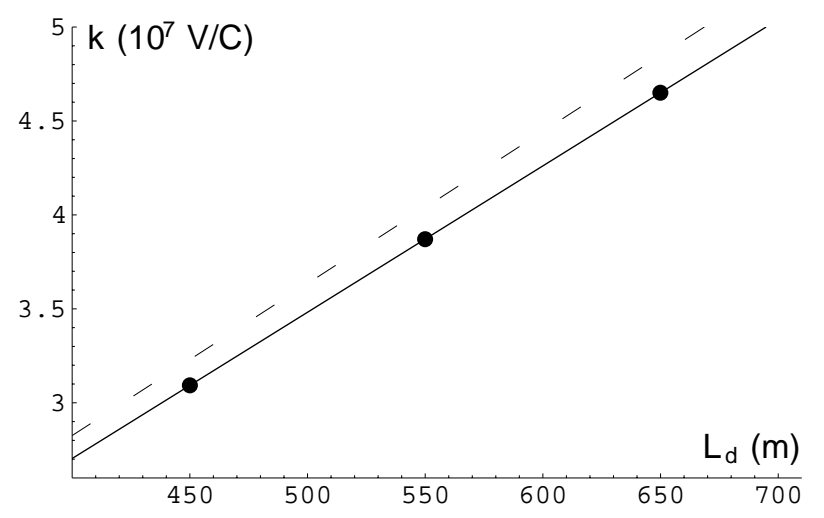

FIG. 3. Loss factor $k$ as a function of the length of the perforated beam screen $L_{d}$ (for a device length $L_{d}$ much longer than the attenuation length $L_{\alpha} \simeq 80 \mathrm{~m}$ ). The solid line refers to Eq. (17) and the dots give the exact numerical values from Eq. (8). The dashed line is obtained neglecting backward wave. We use the following parameters: $b=19 \mathrm{~mm}, d=$ $24.5 \mathrm{~mm}, \rho_{b}=\rho_{d}=7.1 \times 10^{-7} \Omega / \mathrm{m}, D=16 \mathrm{~mm}, \sigma=$ $75 \mathrm{~mm}$, rounded end slots $8 \mathrm{~mm} \times 1.5 \mathrm{~mm}$.

propagating waves in the coaxial, while the solid line also includes the backscattered waves using the approximate formula Eq. (17); the dots give exact numerical values of the loss factor obtained with Eq. (8).

\section{POWER LOST PER UNIT LENGTH}

The excited TEM field dissipates power on the walls of the coaxial region. Inserting Eq. (17) in Eq. (6) yields the specific power loss

$$
P=\frac{\left(\alpha_{m}+\alpha_{e}\right)^{2}}{16 \pi^{4} b^{4} \ln (d / b) S_{b} D}\left[\frac{\sqrt{\pi}}{8} \frac{Z_{0} Q^{2} c^{2}}{\sigma^{3}}+\frac{1}{2} \Gamma\left(\frac{5}{4}\right) \frac{Z_{0} Q^{2} c^{3 / 2}}{a D \sigma^{5 / 2}}+\frac{1}{N} \frac{Z_{0} Q^{2} I}{a^{2} D^{2} c}\right]
$$

A typical behavior of $P$ with the device length $L_{d}$ is drawn in Fig. 4; the dashed curve is the exact value, while the solid lines account for the case without losses and for the limit value reached at long device lengths. The distance between the holes $D$ and their number $N$ is such that the surface covered by the holes is $4.4 \%$ of the total surface of the pipe, as for the LHC liner.

As shown in Fig. 4, there are two simple limiting behaviors of $P$ : it saturates for very long devices, while it grows linearly when the length $L_{d}$ is small.

In the limit of large $L_{d}$, Eq. (18) becomes constant,

$$
P_{\infty}=\lim _{N \rightarrow \infty} P=\frac{\left(\alpha_{m}+\alpha_{e}\right)^{2}}{16 \pi^{4} b^{4} \ln (d / b) S_{b} D}\left[\frac{\sqrt{\pi}}{8} \frac{Z_{0} Q^{2} c^{2}}{\sigma^{3}}+\frac{1}{2} \Gamma\left(\frac{5}{4}\right) \frac{Z_{0} Q^{2} c^{3 / 2}}{a D \sigma^{5 / 2}}\right],
$$

since the number of holes interacting with each other is fixed by the attenuation of the field.

On the contrary, since the field is not dumped efficiently in short lengths, we can use Eq. (15); we get

$$
P_{\operatorname{lin}}=\frac{\sqrt{\pi}}{128 \pi^{4}} \frac{Z_{0} Q^{2} c^{2}}{\sigma^{3}} \frac{\left(\alpha_{m}+\alpha_{e}\right)^{2}}{b^{4} D S_{b} \ln (d / b)} N,
$$

which grows linearly with $N$ (or $L_{d}$ ) because of the interference effect among all the holes.

We define $L_{\alpha}$ as the length where the linear approximation $P_{\text {lin }}$ crosses the limit value $P_{\infty}$; that is,

$$
\begin{aligned}
L_{\alpha} & =D+\frac{4}{\sqrt{\pi}} \Gamma\left(\frac{5}{4}\right) \frac{\sqrt{\sigma}}{a \sqrt{c}} \\
& =D+\frac{4}{\sqrt{\pi}} \Gamma\left(\frac{5}{4}\right) \frac{1}{\alpha\left(\omega_{c}\right)},
\end{aligned}
$$

with $\omega_{c}=2 \pi f_{c}, f_{c}$ is the cutoff frequency of the Gaussian spectrum of the bunch $\left(\omega_{c}=c / \sigma\right)$. We can say approximately that the power per unit length reaches its saturation value for $L>L_{\alpha}$.

$L_{\alpha}$ depends on the attenuation $\alpha$, on the bunch length and on the spacing among the holes. The dependence on 


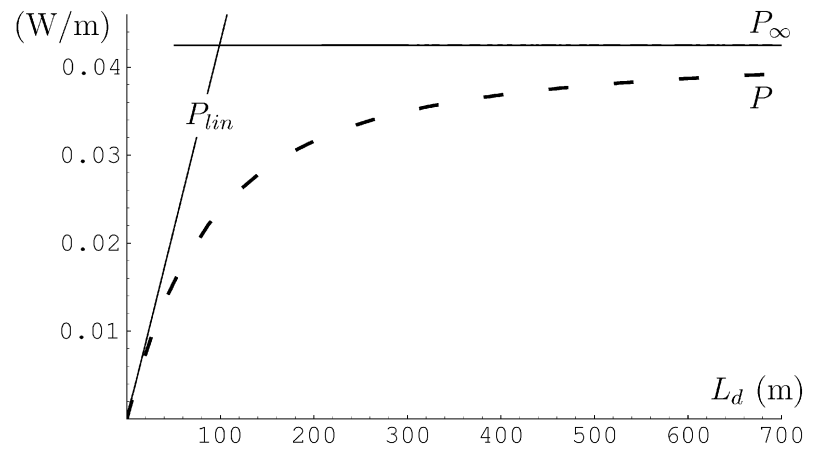

FIG. 4. Power per unit length as a function of the length of the beam screen $L_{d}$. We use the following parameters: $b=$ $19 \mathrm{~mm}, d=24.5 \mathrm{~mm}, \quad \rho_{b}=\rho_{d}=7.1 \times 10^{-7} \Omega / \mathrm{m}, \quad Q=$ $16 \mathrm{nC}, \sigma=75 \mathrm{~mm}$, rounded end slots $8 \mathrm{~mm} \times 1.5 \mathrm{~mm}$. The holes cover a surface of $4.4 \%$ of the total surface of the pipe.

$D$ is not relevant for practical cases (for instance, in the liner of the LHC) because the second term is orders of magnitude bigger. For very strong attenuation (i.e., $\alpha \rightarrow$ $\infty)$, however, the saturation value is reached at lengths equal to the spacing among the holes, as expected. The other term is proportional to the inverse of the attenuation constant computed at the bunch cutoff frequency $f_{c}$; it is not exactly $1 / \alpha\left(\omega_{c}\right)$ (as we could expect) since the loss factor (and $P$ ) is a weighted average on all the frequencies.

If there is more than one hole for a given longitudinal position, we have to multiply our formulas by the number of holes per cross section $N_{b}$ squared. In fact, holes in the same section always interfere constructively, since both the beam field and the TEM field are constant in the azimuthal coordinate and thus they are excited with the same phase.

\section{COMPARISON WITH PREVIOUS RESULTS}

It is interesting to compare our results to those of Ref. [7], where measurements done on a $2 \mathrm{~m}$ long model of the LHC vacuum chamber are reported and interpreted with the help of a simplified model. That paper considers only the forward waves and focuses the attention on the transmission coefficient (i.e., the ratio between the maximum absolute value of the field in the coaxial and the field in the beam pipe) in the two limiting cases of no attenuation (suitable for lengths much smaller than $L_{\alpha}$ ) and of infinite length. The first case is also compared to measurements, showing that the theoretical values of the transmission coefficient are about a factor of 2 below the measured values.

The forward transmission coefficient is given by

$$
G(\omega)=\left|\frac{E_{s r}}{E_{0}}\right|,
$$

where $E_{s r}(z)$ is the electric field radiated by all the holes in the coaxial region and the source field $E_{0}$ is that of a relativistic charge given in Eq. (2). It can be shown [12] that

$$
G(\omega)=\frac{\pi}{\ln (d / b)} \frac{|Z(\omega)|}{Z_{0}} .
$$

Since the absolute value of the coupling impedance is dominated by its imaginary part [given by Eq. (7)], the coefficient $G(\omega)$ becomes

$$
G(\omega) \simeq N \frac{\omega}{4 \pi b^{2} \ln (d / b) c}\left|\alpha_{m}+\alpha_{e}\right| .
$$

This result is identical to the analytical expression derived in [7] written for a thin wall; in fact, when there is no attenuation, the effect of the forward propagating wave (the only wave considered in [7]) dominates.

The saturation value of the power lost per unit length derived in [7] and expressed in our notation (and for a thin wall) is

$$
\hat{P}_{\infty}=\frac{1}{2} \Gamma\left(\frac{5}{4}\right) N_{b}^{2} \frac{Z_{0} Q^{2} c^{3 / 2}\left(\alpha_{m}+\alpha_{e}\right)^{2}}{16 \pi^{4} b^{4} \ln (d / b) S_{b} a D^{2} \sigma^{5 / 2}} .
$$

It is actually the second term of Eq. (19) that is the one accounting for the effect of the forward propagating TEM field, considering $N_{b}$ holes per cross section. In our numerical example, it is the dominating term, since the term accounting for the effect of noninteracting holes and the one due to the backward wave have different signs and almost cancel each other, giving the first term in the square brackets of Eq. (19).

\section{CONCLUSIONS}

We have studied the ideal case of thin wall. Analytical formulas are given for coupling impedance, loss factor, and power lost per unit length for many holes in a coaxial liner and in the presence of attenuation in the coaxial region. To account for finite wall thickness, relevant for practical applications, slight modifications of the present results are needed (see Refs. [5] and [6]) as reported in Appendix B for the reader's convenience.

Interference effects between the holes (not always considered in the literature) are mainly responsible for the beam energy loss. The attenuation in the coaxial region reduces them, but only over a distance comparable to $L_{\alpha}$. These results confirm and generalize previous results; they are also helpful to understand the most relevant physical parameters in the design of the beam screen for real machines.

\section{ACKNOWLEDGMENTS}

The authors are very grateful to S. De Santis, with whom this work started. A.M. wishes to also thank the AP group of the SL Division at CERN for its hospitality during the course of this work. 


\section{APPENDIX A}

To get Eq. (17) inserting Eq. (14) in Eq. (5), we use the following:

$$
\begin{gathered}
\int_{0}^{\infty} \omega^{2} e^{-(\omega \sigma / c)^{2}} d \omega=\frac{\sqrt{\pi}}{4}\left(\frac{c}{\sigma}\right)^{3}, \\
\int_{0}^{\infty} \omega^{3 / 2} e^{-(\omega \sigma / c)^{2}} d \omega=\frac{1}{2} \Gamma\left(\frac{5}{4}\right)\left(\frac{c}{\sigma}\right)^{5 / 2}, \\
I=\int_{0}^{\infty} \omega\left(e^{-a D N \sqrt{\omega}}-1\right) e^{-(\omega \sigma / c)^{2}} d \omega \\
=-\frac{c^{2}}{2 \sigma^{2}}+\frac{c^{2}}{2 \sigma^{2}}{ }_{1} F_{3}\left(1 ; \frac{1}{4}, \frac{1}{2}, \frac{3}{4} ; \frac{a^{4} c^{2}}{256 \sigma^{2}} N^{4} D^{4}\right)-\Gamma\left(\frac{5}{4}\right) \frac{a}{2}\left(\frac{c}{\sigma}\right)^{5 / 2}{ }_{0} F_{2}\left(; \frac{1}{2}, \frac{3}{4} ; \frac{a^{4} c^{2}}{256 \sigma^{2}} N^{4} D^{4}\right) N D \\
+\frac{\sqrt{\pi} a^{2} c^{3}}{8 \sigma^{3}}{ }_{0} F_{2}\left(; \frac{3}{4}, \frac{5}{4} ; \frac{a^{4} c^{2}}{256 \sigma^{2}} N^{4} D^{4}\right) N^{2} D^{2}-\Gamma\left(\frac{3}{4}\right) \frac{a^{3}}{16}\left(\frac{c}{\sigma}\right)^{7 / 2}{ }_{0} F_{2}\left(; \frac{5}{4}, \frac{3}{2} ; \frac{a^{4} c^{2}}{256 \sigma^{2}} N^{4} D^{4}\right) N^{3} D^{3},
\end{gathered}
$$

where ${ }_{p} F_{q}$ is the generalized hypergeometric series [15].

\section{APPENDIX B}

To account for the finite wall thickness, it is necessary to slightly modify all the equations, in particular, Eqs. (18) and (19). The finite thickness changes the problem geometry and, more importantly, introduces an attenuation of the fields in the holes: (i) Denoting by $b_{1}$ and $b_{2}$ the inner and the outer radii of the beam pipe, respectively, one can see that the factor $b^{2}$ in the denominator of all the relations for coupling impedance, loss factor, and forward transmission coefficient has to be replaced by the product $b_{1} b_{2}$; analogously, $\ln (d / b)$ becomes $\ln \left(d / b_{2}\right)$. (ii) The polarizabilities must be corrected; recalling that $\alpha_{e}$ and $\alpha_{m}$ are the thin wall polarizabilities, we can introduce the thick wall polarizabilities $\tilde{\alpha}_{e}$ and $\tilde{\alpha}_{m}$,

$$
\tilde{\alpha}_{e}=\alpha_{e} f(T, \text { hole dimensions }) \quad \text { and } \quad \tilde{\alpha}_{e}=\alpha_{m} g(T, \text { hole dimensions }) .
$$

The functions $f$ and $g$ depend on the hole's shape, but they are always decreasing exponentially with the wall thickness $T$. A first expression for them was given in 1972 by McDonald [16] for circular and rectangular holes. Later, using a variational approach, Gluckstern and Diamond reached an analogous result for circular holes [17]. A first review of McDonald's results and their application to the problem of pumping slots in particle accelerators was presented by Kurennoy [1]; McDonald's results, with minor improvements, have been used in [5] and [6], where they are compared with numerical simulations (MAFIA).

[1] S. Kurennoy, Part. Accel. 39, 1 (1992); Superconducting Super Collider Laboratory Technical Report No. SSCL636 (unpublished).

[2] G. Stupakov, Phys. Rev. E 51, 3515 (1995).

[3] R. Collin, Field Theory of Guided Waves (Oxford University Press, Oxford, 1995), 2nd ed.

[4] S. De Santis, M. Migliorati, L. Palumbo, and M. Zobov, Phys. Rev. E 54, 800 (1996).

[5] S. De Santis, A. Mostacci, and L. Palumbo, Phys. Rev. E 56, 5990 (1997).

[6] S. De Santis, A. Mostacci, L. Palumbo, and B. Spataro, Phys. Rev. E 58, 6565 (1998).

[7] F. Caspers, E. Jensen, and F. Ruggiero, in Proceedings of the European Particle Accelerator Conference, Berlin,
1992, edited by H. Henke, H. Homeyer, and C. Petit-JeanGenaz (Editions Frontières, Gif-sur-Yvette, France, 1992), pp. 889-891.

[8] A. Fedotov and R. Gluckstern, Phys. Rev. E 56, 3583 (1997).

[9] A. Fedotov and R. Gluckstern, Phys. Rev. E 56, 7217 (1997).

[10] L. Palumbo, V. G. Vaccaro, and M. Zobov, in Proceedings of the CERN Accelerator School: Advanced Accelerator Physics Course, Rhodes, 1993, edited by S. Turner (European Laboratory for Particle Physics, Geneva, Switzerland, 1995), pp. 331-390.

[11] A. Chao, Physics of Collective Beam Instabilities in High Energy Accelerators, Wiley Series in Beam Physics and Accelerator Technology (Wiley-Interscience, New York, 1993).

[12] A. Mostacci, CERN LHC Project Report No. 199 (unpublished).

[13] LHC Study Group, CERN Report No. CERN/AC/9505(LHC), 1995.

[14] R. Cooper, in Proceedings of the Cern Accelerator School: RF Engineering for Particle Accelerators, Oxford, $U K, 1991$ (European Laboratory for Particle Physics, Geneva, Switzerland, 1992), pp. 264-265.

[15] I. Gradshtein and I. Ryzhic, Table of Integrals, Series and Products (Academic Press, New York, 1980), p. 1045.

[16] N. A. McDonald, IEEE Trans. Microwave Theory Tech. 20, 689 (1972).

[17] R.L. Gluckstern and J.A. Diamond, IEEE Trans. Microwave Theory Tech. 39, 274 (1991). 